\title{
PREVALÊNCIA DE LESÕES ORAIS EM ESCOLARES DO SUDOESTE DA BAHIA
}

\author{
Maria da Conceição Andrade de Freitas ${ }^{1}$ \\ Claudio Leite de Santana \\ João Vagner Cordeiro Melo ${ }^{3}$ \\ Leila Maria da Silva Batista ${ }^{3}$ \\ Débora Laís Ribeiro e Ribeiro ${ }^{3}$
}

\section{RESUMO}

As patologias orofaciais em crianças são uma realidade em termos de saúde e doença bucal. Neste estudo, os autores avaliaram a prevalência das lesões orais de tecido tegumentar e ósseo em escolares de 06 a 12 anos da rede pública de Jequié-BA, no Brasil. Foram realizados exames clínicos e ortopantomográficos em 209 escolares entre 06 a 12 anos, de ambos os sexos, atendidos no Curso de Odontologia da Universidade Estadual do Sudoeste da Bahia (UESB) entre setembro e dezembro de 2009. Os diagnósticos clínico, radiográfico e histopatológico das lesões encontradas consistem em 09 alterações de desenvolvimento dentário, 04 lesões inflamatórias periapicais, 02 herpes simples, 01 odontoma, 01 displasia cemento-óssea focal e 01 cisto mucoso

\footnotetext{
${ }^{1}$ Mestra em Odontologia na área de concentração de Patologia Buco-Dental, da Faculdade de Odontologia da Universidade da Bahia (FOUFBA). Professora Assistente de Patologia BucoDental e Diagnóstico Oral, do Curso de Odontologia, da Universidade Estadual do Sudoeste da Bahia (UESB). E-mail: conca.freitas@hotmail.com

${ }^{2}$ Mestre em Odontologia área de concentração de Estomatologia da Faculdade de Odontologia da Universidade da Bahia (FOUFBA). Professor Assistente de Cirurgia Bucomaxilo, do Curso de Odontologia da Universidade Estadual do Sudoeste da Bahia (UESB).

${ }^{3}$ Discentes do Curso de Odontologia da Universidade Estadual do Sudoeste da Bahia (UESB).

\begin{tabular}{l|l|l|l|l}
\hline Revista Extensão \& Cidadania & Vitória da Conquista & v. 1, n. 2 & p. 13-20 & jul/dez. 2013 \\
\hline
\end{tabular}
}


do seio maxilar. Neste contexto, é necessário elaborar ações de prevenção, promoção e recuperação da saúde bucal nesta faixa etária.

Palavras-chave: Crianças. Levantamento epidemiológico. Patologias orofaciais.

\begin{abstract}
In this epidemiological study the authors evaluated the prevalence of the oral lesions of soft and bony tissues in children of 6 to 12 years of age of public school, Jequié, BA, Brasil. Clinical and radiograph examination were performed in 209 students between the ages of 6 to 12 years, both sexes, attended at Dentistry Course of Universidade Estadual do Sudoeste da Bahia (UESB), during September until December, 2009. The diagnosed lesions were 09 dental pathologies, 04 periapical pathologies, 02 herpes, 01 odontoma, 01 focalcementum dysplasia and 01 mucous retention cyst. In this context, it's necessary propose specific measures to promote health.
\end{abstract}

Keywords: Children. Epidemology. Oralfacial pathology.

\title{
Introdução
}

Os levantamentos epidemiológicos básicos de saúde oral são usados para coleta de informações sobre as condições orais e as necessidades de tratamento de uma população, e posteriormente, para monitorar as mudanças nos níveis e padrões da doença ao longo do tempo e na avaliação das ações coletivas e individuais, preventivas e assistenciais desenvolvidas (OMS, 1999). As patologias orofaciais em crianças e adolescentes são uma realidade em termos de saúde bucal, entretanto, o levantamento epidemiológico sobre lesões de tecido mole e ósseo encontradas no complexo maxilo-mandibular não é relatado nos programas de saúde pública no Brasil (CAVALCANTE et al., 1999) e, também, pouco relatado na literatura nacional.

Os diversos trabalhos existentes na literatura sobre patologias orofaciais na infância avaliam, por meio de estudo retrospectivo, a prevalência dessas lesões, correlacionando-as com o sexo e idade dos indivíduos. Dentre as citadas, estão as das glândulas salivares, 
cistos da boca, tumores odontogênicos, manifestações bucais de origem infecciosa, viral e fúngica e neoplasias benignas e malignas (GULTELKIN; TOKMAN; TURKSEVEN, 2003; KAMULEGEYA; KALYANYAMA, 2008; LIMA et al., 2008; MAJORANA et al., 2010). Isso demonstra a variedade de lesões tegumentares e ósseas que podem ser encontradas na mucosa bucal e no complexo maxilo-mandibular nesta faixa etária.

No Brasil, os poucos estudos retrospectivos existentes sobre prevalência de patologias orais tegumentares e ósseas em crianças abrangem os estados de Minas Gerais, São Paulo e Rio Grande do Sul. O levantamento da prevalência dessas manifestações bucais nesta faixa etária nas demais regiões do país é bastante relevante, uma vez que podem ser observadas divergências (MAIA et al., 2000; SOUSA et al., 2002; LIMA et al., 2008).

Baseado nestes dados, esse estudo propôs o levantamento epidemiológico das lesões de tecido tegumentar e ósseo do complexo maxilo-mandibular em escolares da rede pública, de 06 a 12 anos de idade, em Jequié, na Bahia, e posteriormente o tratamento e monitoramento das doenças diagnosticadas.

\section{Material e método}

Após aprovação da pesquisa, pelo Comitê de Ética da Universidade Estadual do Sudoeste da Bahia (UESB), foi realizado esse estudo transversal, descritivo e epidemiológico com escolares da rede pública do município de Jequié, na Bahia, no período de 04 meses. A amostra foi composta por 209 estudantes, de ambos os sexos, entre 06 a 12 anos de idade. Foi selecionada esta faixa etária por ser idade-índice recomendada pela Organização Mundial de Saúde (OMS) para avaliar o processo saúde-doença em crianças. Foram excluídos os escolares que não permitiram o exame clínico, os que estavam ausentes nos dias das avaliações, os com dados insuficientes para tabulação e os portadores de má oclusão, cárie dentária e/ou periodontopatia. 
As patologias orofaciais foram avaliadas e diagnosticadas por dois examinadores previamente treinados, após exame clínico intra e extrabucal, por meio de luz artificial e espelho clínico, anamnese e exame ortopantomográfico. O exame histopatológico das lesões e a biopsia das odontoma composto, dispasiacemento-óssea focal e cisto mucoso do seio maxilar foram decisivos para o diagnóstico diferencial e complementação do diagnóstico final, uma vez que a avaliação imagenológica, apenas, não possibilitou o diagnóstico.

Após a determinação do diagnóstico oral, os diferentes tipos de lesões encontradas foram correlacionados com a faixa etária e a localização anatômica.

\section{Resultados}

Dos 208 escolares de 06 a 12 anos de idade que foram avaliados num período de 04 meses, obteve-se um total de 15 pacientes com 18 lesões orais diagnosticadas, sendo 03 pacientes portadores de 02 lesões orais. Todas as patologias avaliadas foram de caráter benigno.

As dezoito lesões orais diagnosticadas em ordem decrescente foram: 09 alterações de desenvolvimento dentário, 04 lesões inflamatórias periapicais, 02 herpes simples, 01odontoma, 01 displasia cemento-óssea focal e 01 cisto mucoso do seio maxilar (tabela 1).

Tabela 1 - Listagem de lesões orais diagnosticadas.

\begin{tabular}{l|c|c}
\hline Lesões Orais & $\mathbf{N}^{0}$ de Casos & Percentagem \\
\hline Alterações de Desenvolvimento Dentário & $\mathbf{9}$ & $\mathbf{5 0 \%}$ \\
\hline Lesões Inflamatórias Periapicais & 4 & $\mathbf{2 2 . 2 2 \%}$ \\
\hline Herpes simples & 2 & $\mathbf{1 1 . 1 1 \%}$ \\
\hline Odontoma & $\mathbf{1}$ & $\mathbf{5 . 5 5 \%}$ \\
\hline Displasia Cemento-óssea focal & $\mathbf{1}$ & $\mathbf{5 . 5 5 \%}$ \\
\hline Cisto Mucoso do Seio Maxilar & 1 & $\mathbf{5 . 5 5 \%}$ \\
\hline
\end{tabular}


A faixa etária de maior ocorrência das patologias orais diagnosticadas foi de 08 a 10 anos, com um caso de alteração de desenvolvimento dentário em uma criança de 06 anos de idade (tabela 2).

Tabela 2 - Distribuição das lesões orais em relação à faixa etária.

\begin{tabular}{l|c|c|c}
\hline \multicolumn{1}{c|}{ Lesões Orais } & $\begin{array}{c}6-7 \text { anos } \\
\text { de idade }\end{array}$ & $\begin{array}{c}8-9 \text { anos } \\
\text { de idade }\end{array}$ & $\begin{array}{c}\text { 10-12 anos } \\
\text { de idade }\end{array}$ \\
\hline Alterações de Desenv. Dent. & 1 & 6 & 2 \\
\hline Lesões Inf. Periap. & & 2 & 2 \\
\hline Herpes simples & & 2 & 1 \\
\hline Odontoma & & 1 & \\
\hline Displasia Cemento-óssea focal & & 1 & \\
\hline Cisto Mucoso do Seio Maxilar & & 1 & \\
\hline
\end{tabular}

Fonte: Dados coletados pelos autores.

A distribuição anatômica das lesões orais diagnosticadas está demonstrada na tabela 3. As alterações de desenvolvimento dentário foram mais prevalentes na maxila, enquanto as lesões inflamatórias periapicais, na mandíbula.

Tabela 3 - Distribuição anatômica das lesões orais diagnosticadas.

\begin{tabular}{l|c|c|c|c|c|c|c}
\hline \multirow{2}{*}{ Lesões Orais } & \multicolumn{6}{c}{ Regiões da cavidade oral } \\
\cline { 2 - 7 } & $\begin{array}{c}\text { Antero } \\
\text { superior }\end{array}$ & $\begin{array}{c}\text { Lábio } \\
\text { superior }\end{array}$ & $\begin{array}{c}\text { Antero } \\
\text { inferior }\end{array}$ & $\begin{array}{c}\text { Pré-molar } \\
\text { inferior }\end{array}$ & $\begin{array}{c}\text { Molar } \\
\text { inferior }\end{array}$ & $\begin{array}{c}\text { Seio } \\
\text { maxilar } \\
\text { esquerdo }\end{array}$ & $\begin{array}{c}\text { Comissura } \\
\text { Labial }\end{array}$ \\
\hline $\begin{array}{l}\text { Alterações de } \\
\text { Desenvolvimento } \\
\text { Dentário }\end{array}$ & 5 & & 1 & 3 & & & \\
\hline $\begin{array}{l}\text { Lesões } \\
\text { Inflamatórias } \\
\text { Periapicais }\end{array}$ & & & & 1 & 3 & & \\
\hline Herpes simples & & 1 & & & & & 1 \\
\hline Odontoma & & & & 1 & & & \\
\hline $\begin{array}{l}\text { Displasia } \\
\text { Cemento-óssea } \\
\text { focal }\end{array}$ & & & & & 1 & & \\
\hline $\begin{array}{l}\text { Cisto Mucoso do } \\
\text { Seio Maxilar }\end{array}$ & & & & & & 1 & \\
\hline
\end{tabular}

Fonte: Dados coletados pelos autores. 


\section{Discussão}

No presente estudo, diagnosticamos dezoito lesões orais, sendo $16 \%$ equivalentes às biopsias realizadas. Dhanuthai, Banrai e Limpanaputtajak (2007) relataram $15.05 \%$ das patologias orais biopsiadas dos 1251 casos na faixa etária de 0-16 anos. Esse resultado é ligeiramente mais elevado que o encontrado na literatura, a qual relata um percentual menor que $10 \%$ na infância (MAIA et al., 2000; GULTELKIN; TOKMAN; TURKSEVEN, 2003; JONES; FRANKLIN, 2006; LIMA et al., 2008). A disparidade entre os diferentes estudos pode ser decorrente do critério de inclusão. Alguns estudos abrangem uma faixa etária de 0 até 16 anos. Além disso, outros fatores são relevantes como o período durante o qual a pesquisa foi realizada a região geográfica, a herança genética da população, e a instituição na qual o estudo foi obtido (DHANUTHAI; BANRAI; LIMPANAPUTTAJAK, 2007).

Recentemente, Lima e colaboradores (2008) fizeram um estudo retrospectivo sobre patologias orofaciais biopsiadas, compreendendo um período de 20 anos. No geral, os resultados encontrados nesse estudo corroboram com os relatados pela literatura no que se refere à mucocele e às lesões inflamatórias reacionais como as mais prevalentes na infância. Segundo Dhanuthai, Banrai e Limpanaputtajak (2007) e Maia e colaboradores (2000), as lesões císticas foram as mais comuns. Nossos dados não estão de acordo com a literatura, uma vez que observamos as alterações de desenvolvimento dentário e as patologias inflamatórias periapicais como as mais prevalentes. Também verificamos que todas as lesões orais biopsiadas foram benignas. Esses dados se assemelham aos relatados na literatura, a qual afirma que a maioria das patologias diagnosticadas apresentou benignidade, com um percentual em média de 1\% para as malignas. Contrapondo esses dados, Kamulegeya e Kalyanyama (2008) relataram que 67.28\% dos 1298 casos diagnosticados foram de caráter maligno.

Os diversos estudos epidemiológicos existentes na literatura sobre lesões orais na infância englobam diferentes grupos numa faixa etária que varia de 0 a 16 anos, o que dificulta determinar em qual grupo ocorre essa maior prevalência (DHANUTHAI; BANRAI; 
LIMPANAPUTTAJAK, 2007). A maioria dos trabalhos existentes na literatura relata uma maior prevalência dessas patologias nos grupos mais velhos, entre 11 a 16 anos de idade (JONES; FRANKLIN, 2006; LIMA et al., 2008; PALACIOS et al., 2008). Nesse estudo, os resultados obtidos demonstraram que ocorreu uma maior concentração de lesões orais diagnosticadas na dentição mista.

Majorana et al. (2010), em um estudo retrospectivo de dez anos, avaliaram clinicamente um grupo de crianças de 0 a 12 anos, saudáveis e com doenças crônicas, e encontraram prevalência maior de candidíase, lesões traumáticas, UAR e infecção recorrente por HSV-1, lesões estas que pouco foram observadas na amostra de mesma faixa etária por nós estudada, possivelmente pela heterogeneidade étnica de nossa população.

É de grande relevância salientar que nossos dados foram obtidos com base em uma avaliação de caráter transversal com uma amostra reduzida e com critérios de inclusão divergentes dos demais estudos existentes na literatura.

\section{Conclusão}

Este estudo proporciona informações importantes e alguns pontos de deficiência. Um deles está relacionado ao conhecimento do perfil epidemiológico das patologias orofaciais na infância que, por meio dos exames clínicos, imagenológicos e histopatológicos nas diversas áreas geográficas, não permite ao cirurgião-dentista confirmar ou excluir qualquer hipótese diagnóstica. Outro, é que as lesões foram diagnosticadas após um único exame de cada paciente, provavelmente subestimando a ocorrência de lesões recorrentes como as infecções pelo HSV. Entretanto, tais conhecimentos nos orientam de forma decisiva no processo de diagnóstico e tratamento dessas lesões.

\section{Referências}

CAVALCANTE, A. C. et al. Lesões bucais de tecido mole e ósseo em crianças e adolescentes. Rev. da Fac. de Odontol., São José dos Campos, v. 2, n. 1, p. 67-73, jan./jun. 1999. 
DHANUTHAI, K.; BANRAI, M.; LIMPANAPUTTAJAK. A retrospective study of paediatric lesions from Thailand. International Journal of Paediatric Dentistry, v. 17, p. 248-253, 2007.

GULTELKIN, S. E.; TOKMAN, B.; TURKSEVEN, M. R. A review of paediatric oral biopsies in Turkey. Internacional Dental Journal, v. 53, p. 26-32, 2003.

JONES, A. V.; FRANKLIN, C. D. An analysis of oral and maxillofacial pathology found in children over a 30 -year period. International Journal of Paediatric Dentistry, v. 16, p. 19-30, 2006.

KAMULEGEYA, A.; KALYANYAMA, M. Oral maxillofacial neoplasms in an East African population a 10 year retrospective study of 1863 cases using histopathological reports. Bio Med Central Oral Health, v. 8, n. 19, p. 1-11, 2008.

LIMA, G. S. et al. A survey of oral and maxillofacial biopsies in children. A single-center retrospective study of 20 years in Pelotas-Brasil. Journal of Applied Oral Science, v. 16, n. 6, p. 397-402, 2008.

MAIA, D. M. et al. A survey of oral biopsies in Brazilian pediatric patients. ASDC Journal of Dentristy for Children, v. 67, n. 2, p. 128-131, 2000.

MAJORANA, A. et al. Oral mucosal lesions in children from 0 to 12 years old: ten years' experience. Oral Surg Oral Med Oral Pathol Oral Radiol Endod, v. 110, n. 1, p. 13-8, 2010.

ORGANIZAÇÃO MUNDIAL DE SAÚDE (OMS). Levantamento básico em saúde bucal. Manual de instruções. 4. ed. Santos, 1999.

PALACIOS, J. C. et al. Identification de entidades patológicas em los tejidosblandos bucales de La poblacion rural infantil y adolescente de La unidad educativa padre Luis Ormieres, estado monagas em el período agosto-noviembro 2005. Acta Odontológica Venezolana, v. 46, n. 1, p. 1-8, 2006.

SOUSA, F. B. et al. Pediatric oral lesions: a 15-year review from São Paulo, Brasil. Journal Clinical Pediatric Dental, v. 26, n. 4, p. 413-418, 2002. 